\title{
GW190412: Gravitational wave from an unequal mass binary black hole with precession
}

\author{
ShiChao $\mathrm{Wu}$, and ZhouJian Cao ${ }^{*}$ \\ Department of Astronomy, Beijing Normal University, Beijing 100875, China
}

Received May 20, 2020; accepted August 6, 2020; published online October 23, 2020

Citation: $\quad$ S. C. Wu, and Z. J. Cao, GW190412: Gravitational wave from an unequal mass binary black hole with precession, Sci. China-Phys. Mech. Astron. 63, 129532 (2020), https://doi.org/10.1007/s11433-020-1608-8

The upgraded Advanced LIGO and Advanced Virgo began their third observing run (O3) on April 1, 2019. This observation, however, was suspended on March 27, 2020 owing to the impact of the COVID-19 pandemic, leaving the follow-up arrangements undetermined.

The LIGO Scientific Collaboration and Virgo Collaboration (LVC) has so far announced 56 gravitational wave candidates on the GraceDB website, coupled with two gravitational wave events analyzed in detail [1,2]. GW190425, the first gravitational wave event announced in $\mathrm{O} 3$, is likely the second observed gravitational wave event of a binary neutron star (BNS) inspiral, with its distance much farther than that of GW170817 [3]. GW190412, the second gravitational wave event announced in $\mathrm{O} 3$, actually happened earlier than GW190425 but took a longer time for data analysis due to its complexity. Compared with other GW candidates, GW190412 has several unique characteristics, including the large mass ratio, the higher-order mode clearly evidenced [4], and the strongest constraint on precession and primary black hole's spin observed so far.

In the first two observing runs $(\mathrm{O} 1 / \mathrm{O} 2), 10$ binary black hole (BBH) gravitational wave events that are classified into the first Gravitational-Wave Transient Catalog (GWTC-1) by LVC share a common feature, that is, the black holes in the binary system have comparable masses [5]. But this trend was broken by the GW event of the binary black hole ob-

"Corresponding author (email: zjcao@amt.ac.cn) served on April 12, 2019 at 05:30:44 UTC. This gravitational wave signal GW190412 was jointly detected by two Advanced LIGO detectors and one Advanced Virgo detector. The signal-to-noise ratio of the detector network is 19 , and the false alarm rate (FAR) is less than 1 per $10 \times 10^{5}$ years by the GstLAL pipeline. Bayesian parameter estimation indicates that GW190412 was generated by the merger of two black holes respectively with 30 times solar mass and 8 times solar mass. According to the prediction of the general relativity, GW signals produced by a BBH with a high massratio are expected to have higher-order modes. This prediction has been confirmed by the observation of GW190412 (Figure 1).

In the context of GW data analysis, the luminosity distance and inclination angle of the source binary are usually degenerated, so it is usually difficult to accurately measure both parameters. This degeneracy may be changed by the existence of higher-order modes [2], for instance in GW190412. When using the binary black hole gravitational wave template containing higher-order modes for parameter estimation, the posterior probability distribution obtained (the green line) is significantly better than the result without higher-order modes (the blue line), breaking the V-shaped distribution. More interestingly, the posterior distribution of these two parameters is further narrowed by precession (the orange line).

Both the effective-one-body numerical relativity (EOBNR) templates and phenomenological model (Phenom) 


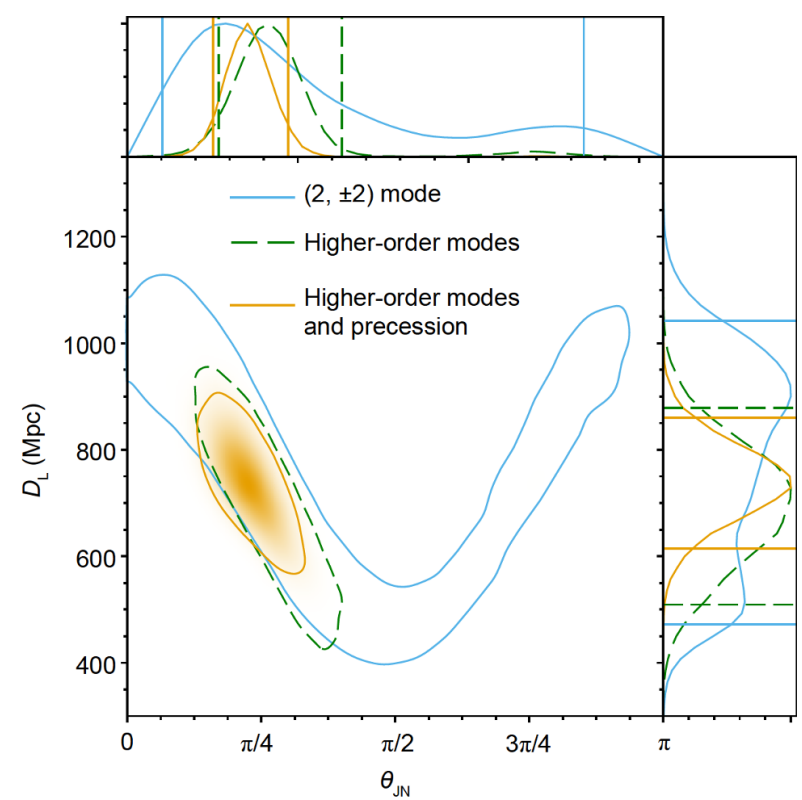

Figure 1 (Color online) The posterior probability distribution $(90 \%$ credible regions) of luminosity distance and inclination angle for GW190412. When using a BBH waveform template with higher-order modes and precession, the posterior probability distribution of these two parameters is best constrained. This posterior probability distribution plot is drawn with the public data of GW190412 from https://dcc.ligo.org/LIGOP190412/public.

templates are used by LVC in data analyses [2]. For these two kinds of waveform approximants, the templates with dominant-multipole non-precession, higher multipole (higher-order modes) non-precession, and higher multipole precession have been used in data analyses. The template with both higher multipole and precession got the largest signal-tonoise ratio.

LVC adopted four independent data analysis methods to confirm the higher-order mode hypothesis for GW190412 [2]. The first method is the standard parameter estimation method that compares Bayes factors of different templates and the matched filtering signal-to-noise ratio. The resulting Bayes factors support the existence of precession and higherorder modes. The second method is to calculate the optimal signal-to-noise ratio for the individual mode. The entire analysis was performed with the IMRPhenomHM template [6]. The results show that the $(3, \pm 3)$ mode is the strongest mode among higher-order modes. The third method is to calculate the signal energy of individual mode in the timefrequency domain [7] with an empirical formula relating the gravitational wave frequencies of higher-order modes to that of $(2, \pm 2)$ mode $f_{l m}(t) \approx \alpha f_{22}(t)$. Gravitational waves of different modes (indicated by different $\alpha=m / 2$ ) can map different tracks on the time-frequency plot, and the summed signal energy on each track can reflect the energy of different modes. Based on a fixed time-frequency resolution, the signal energy of $\alpha=1$ and $\alpha=1.5$ is demonstrated to be the highest, corresponding to the $(2, \pm 2)$ mode and $(3, \pm 3)$ mode, respectively. The fourth method is to calculate the fitting factor between the wavelet-based reconstructed waveform and different waveform templates. Fitting the dominant mode non-precession template, higher-order mode non-precession template and higher-order mode precession template compared respectively to the reconstructed waveform through BayesWave [8], it is found that the templates with both precession and higher-order modes match the waveletbased reconstructed waveform best. All the four methods show that GW190412 has the higher-order mode and precession.

According to Kullback-Leibler divergence [9], GW190412 offers the strongest evidence for the precession of all BBHs detected so far, $\chi_{p} \in[0.15,0.49]$ at $90 \%$ probability. The asymmetric masses of GW190412 indicate that the spin of a more massive $\mathrm{BH}$ dominates the spin contribution. For the first time, we can measure the spin of a component $\mathrm{BH}$, $\chi_{1}=0.43_{-0.26}^{+0.16}$ at $90 \%$ probability. We can use spins to understand the formation channel of BBHs detected by LIGO and Virgo, because spins of BBHs formed in the isolated binary channel and dynamical formation channel will be different [10].

In summary, GW190412 is the first BBH event demonstrating the existence of the higher-order mode and precession. And for the first time, we can measure the spin of a black hole. With more and more GW events to be observed in the near future, we will clarify whether the GW190412-like events are common.

1 B. P. Abbott, et al. (LIGO Scientific Collaboration and Virgo Collaboration), Astrophys. J. Lett. 892, L3 (2020).

2 R. Abbott, et al. (LIGO Scientific Collaboration and Virgo Collaboration), Phys. Rev. D 102, 043015 (2020).

3 H. Gao, Sci. China-Phys. Mech. Astron. 61, 059531 (2018).

4 M. Ruiz, M. Alcubierre, D. Núñez, and R. Takahashi, Gen. Relativ. Gravit. 40, 1705 (2008), arXiv: 0707.4654.

5 B. P. Abbott, et al. (LIGO Scientific Collaboration and Virgo Collaboration), Phys. Rev. X 9, 031040 (2019), arXiv: 1811.12907.

6 L. London, S. Khan, E. Fauchon-Jones, C. García, M. Hannam, S. Husa, X. Jiménez-Forteza, C. Kalaghatgi, F. Ohme, and F. Pannarale, Phys. Rev. Lett. 120, 161102 (2018), arXiv: 1708.00404.

7 S. Roy, A. S. Sengupta, and K. G. Arun, arXiv: 1910.04565.

8 N. J. Cornish, and T. B. Littenberg, Class. Quantum Grav. 32, 135012 (2015), arXiv: 1410.3835 .

9 S. Kullback, and R. A. Leibler, Ann. Math. Statist. 22, 79 (1951).

10 B. Farr, D. E. Holz, and W. M. Farr, Astrophys. J. 854, L9 (2018), arXiv: 1709.07896. 